\section{(6) \\ OPEN ACCESS}

\title{
Patient understanding of liver cirrhosis and improvement using multimedia education
}

\author{
Matthew A Goldsworthy, ${ }^{1}$ Waleed Fateen, ${ }^{1,2}$ Helene Thygesen, ${ }^{3}$ \\ Mark A Aldersley, ${ }^{4}$ Ian A Rowe, ${ }^{1,4}$ Rebecca L Jones ${ }^{4}$
}

- Additional material is published online only. To view please visit the journal online (http://dx doi.org/10.1136/ flgastro-2016-100761)

${ }^{1}$ School of Medicine, University of Leeds, Leeds, UK

${ }^{2}$ NIHR Digestive Diseases

Biomedical Research Unit,

Nottingham University Hospitals NHS Trust and The University of Nottingham, Nottingham, UK

${ }^{3}$ College of Health and Wellbeing, University of Central

Lancashire, Preston, UK ${ }^{4}$ Department of Hepatology, Leeds Teaching Hospitals NHS Trust, Leeds, UK

Correspondence to Dr Waleed Fateen, NIHR Nottingham Digestive Diseases Biomedical Research Unit, Queens Medical Centre, Derby Road, Nottingham NG7 2UH, UK; waleed.fateen@nottingham. ac.uk

Received 18 October 2016 Revised 26 December 2016 Accepted 1 January 2017

Published Online First

19 January 2017

CrossMark

To cite: Goldsworthy MA, Fateen $\mathrm{W}$, Thygesen $\mathrm{H}$, et al. Frontline Gastroenterology 2017:8:214-219.

\section{ABSTRACT}

Objective For patients to engage with the longterm management of liver cirrhosis, sufficient understanding of their condition is essential. The aim of this study was to assess baseline patient knowledge and to test whether a conditionspecific multimedia screencast could improve this.

Design Service quality improvement study. Setting A UK tertiary liver centre. Patients were recruited during 12 general hepatology outpatient clinics.

Patients Fifty-two patients with liver cirrhosis were included. Sixty-two per cent were male; their median age was 56 years and their median clinic attendance period was 3 years.

Interventions Participants completed a baseline questionnaire assessing their knowledge of the management and complications of cirrhosis. They then watched a tailored screencast discussing this condition, which had been developed by expert hepatologists in collaboration with patient representatives. Knowledge was reassessed using a new copy of the original questionnaire after an interval of at least one month.

Main outcome measures Patient scores on knowledge questionnaires at baseline and follow-up

Results Fifty-two patients achieved a median score of $25.0 \%$ before viewing the screencast. Thirty-five patients then completed a follow-up questionnaire after an interval period. The median questionnaire score in this group improved from $25.0 \%$ to $66.7 \%$; an increase of $41.7 \%$ compared with baseline $(p<0.001)$.

Conclusions Despite regular review at a specialist clinic, participants had poor baseline knowledge of liver cirrhosis. Delivering information by screencast led to a significant improvement. We therefore present an effective way to empower patients with accurate, up-to-date and retainable information that can easily be translated to many other conditions.

\section{INTRODUCTION}

Chronic liver disease mortality is increasing in the UK, and the number of people living with the condition also continues to rise. ${ }^{1}$ Those individuals with cirrhosis have an appreciable annual risk of developing hepatocellular carcinoma (HCC), of liver decompensation-including variceal haemorrhage-and of liver-related mortality. ${ }^{2}$ It is therefore essential that these patients engage with ongoing management, especially surveillance for the early detection of HCC and oesophageal varices, as these are seen as primary methods to reduce mortality in this population.

Such patient engagement is dependent upon individuals having sufficient understanding of their condition and its management to allow them to actively participate in care and make decisions. ${ }^{3}$ Presently, however, patient understanding may be limited. ${ }^{5}$ Reasons for this include the restricted time available for counselling during clinic consultations and the variable quality of educational resources such as websites and information leaflets. ${ }^{6}$ The inherent complexity of liver cirrhosis and its complications may also restrict some patients in achieving sound understanding. In order for patients to continually engage with long-term management, however, it is essential that effort be made to facilitate them developing this understanding. Indeed, patient education has recently been identified as a key unmet need in the supportive care of patients with chronic liver disease. ${ }^{7}$ 
Despite this, little evidence exists regarding patient understanding of cirrhosis or the effectiveness of a range of educational tools routinely used in practice. One study was identified in which patients' knowledge of cirrhosis self-management was tested at a single centre in the USA. ${ }^{8}$ This found poor baseline knowledge that significantly improved after patients were issued with an information booklet.

In this study, we sought to assess the baseline knowledge of a cohort of patients with liver cirrhosis at a major UK centre. We then sought to test the effectiveness of an adjunct to traditional counselling: a condition-specific multimedia screencast. This is a narrated video that presents relevant and evidence-based information about liver cirrhosis and its management, supported by on-screen text, diagrams and animations. The use of alternative formats to written information has been shown to rate favourably in terms of patient satisfaction and may also increase the accessibility of the information to a wider range of patients. ${ }^{3}$ Clinical outcomes may also benefit from this approach. For example, using a video to supplement verbal counselling and written information was shown to significantly improve attendance for mammography screening. ${ }^{9}$ If such an intervention were to be effective in improving patient understanding of liver cirrhosis, it could be used in routine practice as an educational tool. To our knowledge, this is the first time that such an intervention has been evaluated in patients with liver disease.

\section{METHODS}

We produced a screencast entitled 'Understanding Liver Cirrhosis'. This was developed in collaboration with consultant hepatologists to ensure its accuracy and relevance. It was also reviewed by patient representatives to ensure suitability for a lay audience. The 12-min long video explains the functions of the liver, the development of cirrhosis as progression through inflammation and scarring due to exposure to a causative factor, the complications that may arise from this and the associated management strategies. Figure 1 shows a screenshot from the video in which a schematic illustration is being used to explain the development of oesophageal varices and the need for endoscopic surveillance. To maximise accessibility, simple language was used throughout, supported by subtitles, images and animations. Signposting, repetition and summarisation techniques were also used to aid patient understanding. The full screencast is available to view online (https://goo.gl/bRd2pH).

We designed a questionnaire with which to assess patient knowledge of the complications and management of liver cirrhosis at baseline, and then again after viewing the screencast. An initial draft was assessed for face validity by two members of a patient support group and three patient liaison staff members. Improvements to clarity and structure were made in response to feedback. Content validity was established through consultation with two consultant hepatologists with expertise in cirrhosis management.

The final version (see online supplementary file 1) consisted of six single-best-answer questions, one multiple-choice question and one short-written-answer question. The single-best-answer questions had a two-part structure. The first asked whether the participant knew the reason for a particular complication or management strategy, to which they could answer yes or no. If they answered yes, the second part offered them three reasons to choose from, along with a fourth 'other' option that could be written in a box provided. This structure was intended to best represent participants' understanding by discouraging guessing if they did not know the answer and by providing space for them to give a different response if they felt that none of the options given was suitable. The questions assessed participants' knowledge of the reasons for having ultrasound scans, endoscopies and bone mineral density scans; their knowledge of the causes of oesophageal varices and hepatic encephalopathy; their understanding of the reasons for taking laxatives; their awareness of muscle wasting, coagulopathy and liver cancer as being complications of cirrhosis (multiplechoice question) and their knowledge of the functions of the liver (short-written-answer question).

Patients with liver cirrhosis attending a general hepatology outpatient clinic who were aged $\geq 18$ years and on a surveillance programme for HCC were eligible to participate. Those who were not aware they had liver cirrhosis were excluded. The medical records of patients scheduled to attend 12 outpatient clinics between September 2013 and January 2014 were assessed. Eligible patients were approached on arrival for their appointment. The study was approved as a service development project by the department in which the work was conducted. Informed consent was obtained from all patients.

Participants first completed a baseline questionnaire to assess their knowledge of the complications and management of liver cirrhosis. They then watched the screencast on a consultation room computer screen, accompanied, if desired, by a relative or supporter. Viewers were then asked to evaluate the concept and quality of the screencast. A new copy of the knowledge questionnaire was sent to patients through the post after an interval of at least one month (range 16 months). They were asked to complete this without the help of resources or other people and to return it by post. We attempted to remind patients who failed to reply by telephone and offered them the chance to complete the questionnaire online.

Questionnaires were marked by allocating one point for a correct answer and no points for any other response, such as an incorrect answer, no answer or multiple options selected. Statistical analysis was conducted using SPSS (V.22.0, IBM, Chicago, Illinois, 


\section{Enlarged veins in the oesophagus}

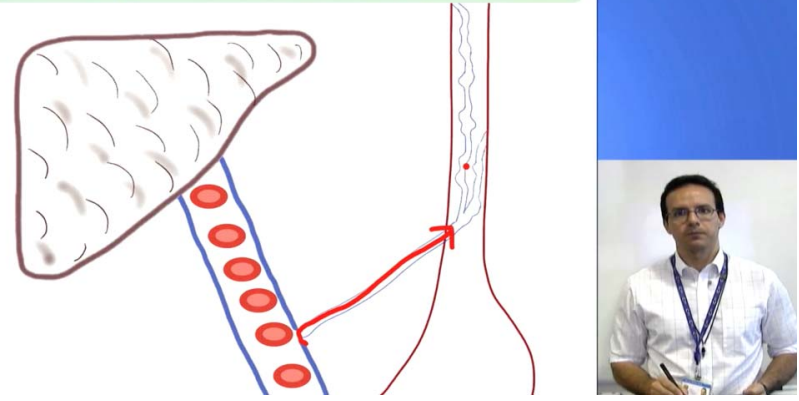

Figure 1 An image from the screencast in which the presenter (WF) draws a schematic illustration to explain the development of oesophageal varices in liver cirrhosis.

USA). Due to skewed distributions, medians and IQRs are reported as summary measures. The Wilcoxon signed-rank test was used for paired comparisons of overall questionnaire scores between baseline and follow-up. The McNemar test was used to compare the proportions of correct responses to individual questions between baseline and follow-up. p $<0.05$ was considered statistically significant.

\section{RESULTS}

Fifty-eight patients were identified from medical records as being eligible for inclusion. Five of these answered 'no' when asked if they had ever been told they had liver cirrhosis and were therefore excluded. One patient declined to participate due to being

Table 1 Demographic data for patients who completed the baseline and follow-up questionnaires

\begin{tabular}{|c|c|c|c|}
\hline & \multirow[b]{2}{*}{ Baseline $(n=52)$} & \multicolumn{2}{|c|}{ Follow-up questionnaire } \\
\hline & & Yes $(n=35)$ & No $(n=17)$ \\
\hline \multicolumn{4}{|l|}{ Gender-n (\%) } \\
\hline Male & $32(61.5 \%)$ & $22(62.9 \%)$ & $10(58.8 \%)$ \\
\hline Female & $20(38.5 \%)$ & $13(37.1 \%)$ & $7(41.2 \%)$ \\
\hline $\begin{array}{l}\text { Age, years—median } \\
(\mathrm{IQR})\end{array}$ & $55.5(20.5)$ & $62.0(15.0)$ & $48.0(17.0)$ \\
\hline $\begin{array}{l}\text { Clinic attendance } \\
\text { period, years- } \\
\text { median (IQR) }\end{array}$ & $3.0(2.0)$ & $3.0(2.0)$ & $3.0(2.5)$ \\
\hline \multicolumn{4}{|l|}{ Diagnosis-n (\%) } \\
\hline $\begin{array}{l}\text { Alcohol-related } \\
\text { liver disease }\end{array}$ & $19(36.5 \%)$ & $12(34.3 \%)$ & $7(41.2 \%)$ \\
\hline Viral hepatitis & $10(19.2 \%)$ & $8(22.9 \%)$ & $2(11.8 \%)$ \\
\hline $\begin{array}{l}\text { Non-alcoholic } \\
\text { fatty liver disease }\end{array}$ & $9(17.3 \%)$ & $8(22.9 \%)$ & $1(5.9 \%)$ \\
\hline $\begin{array}{l}\text { Cholestatic liver } \\
\text { disease }\end{array}$ & $9(17.3 \%)$ & $5(14.3 \%)$ & $4(23.5 \%)$ \\
\hline Other & $5(9.6 \%)$ & $2(5.7 \%)$ & $3(17.6 \%)$ \\
\hline $\begin{array}{l}\text { Reported previous } \\
\text { endoscopy—n (\%) }\end{array}$ & $43(82.7 \%)$ & $28(80.0 \%)$ & $15(88.2 \%)$ \\
\hline $\begin{array}{l}\text { Reported previous } \\
\text { liver ultrasound—n } \\
(\%)\end{array}$ & $48(92.3 \%)$ & $31(88.6 \%)$ & $17(100 \%)$ \\
\hline
\end{tabular}

frightened of knowing more about cirrhosis. The remaining 52 patients provided informed consent and were entered into the study. The characteristics of this cohort are detailed in table 1 . The median clinic attendance period was 3 years (range 1 month to 13 years). More than $80 \%$ of participants had undergone upper gastrointestinal endoscopy as screening for oesophageal varices and more than $90 \%$ had undergone abdominal ultrasound scanning as surveillance for HCC. For patients who recently began attending this clinic, these procedures had not been performed by the time of inclusion in this study, although relevant counselling had been provided at previous appointments.

\section{Baseline knowledge}

When asked to identify complications of cirrhosis in a multiple-choice question, 8 of the 52 participants $(15 \%)$ correctly selected liver cancer, impaired coagulation and muscle wasting; a further eight patients did not select any of these. Only $25 \%$ of patients were aware that there was a risk of internal bleeding due to oesophageal varices and just $17 \%$ understood that there was a liability to develop hepatic encephalopathy due to impaired clearance of toxins. Four patients $(8 \%)$ were aware that laxatives were prescribed to help remove such toxins through the bowel.

Regarding surveillance investigations, of the 48 patients reporting that they had previously undergone an ultrasound scan of the liver, only $5(10 \%)$ correctly identified cancer screening as the rationale for regular scanning in liver cirrhosis. Similarly, of the 43 patients reporting a previous upper gastrointestinal endoscopy, only $23(53 \%)$ correctly identified screening for oesophageal varices as the purpose of this investigation. Also, 30 patients reported a previous bone mineral density scan, of which $20(67 \%)$ knew that the purpose of this was to detect bone weakening.

Overall, the median baseline questionnaire score for the cohort was 25.0\% (IQR 16.7-41.7\%). Figure 2 presents the median scores in each aetiology subgroup. Patients with non-alcoholic fatty liver disease 


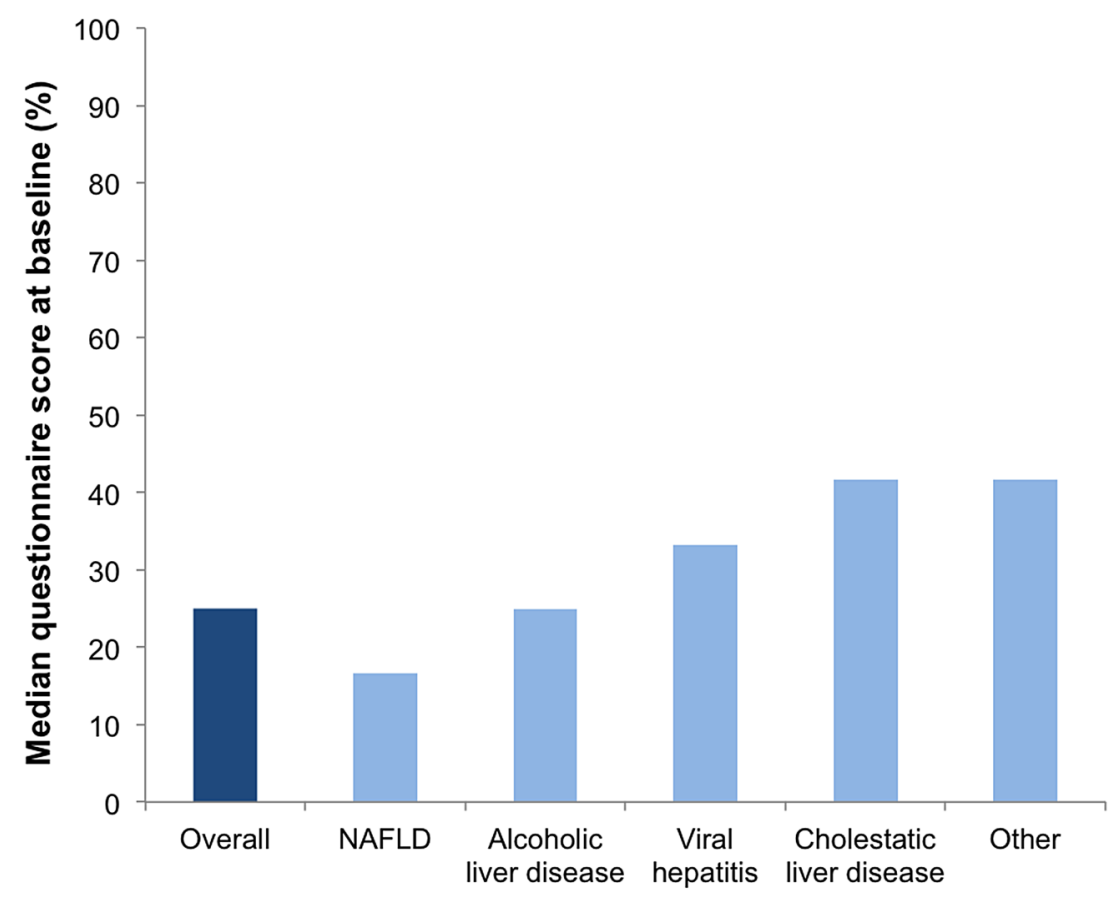

Aetiology of cirrhosis

Figure 2 Median baseline questionnaire scores grouped by cirrhosis aetiology. NAFLD, non-alcoholic fatty liver disease.

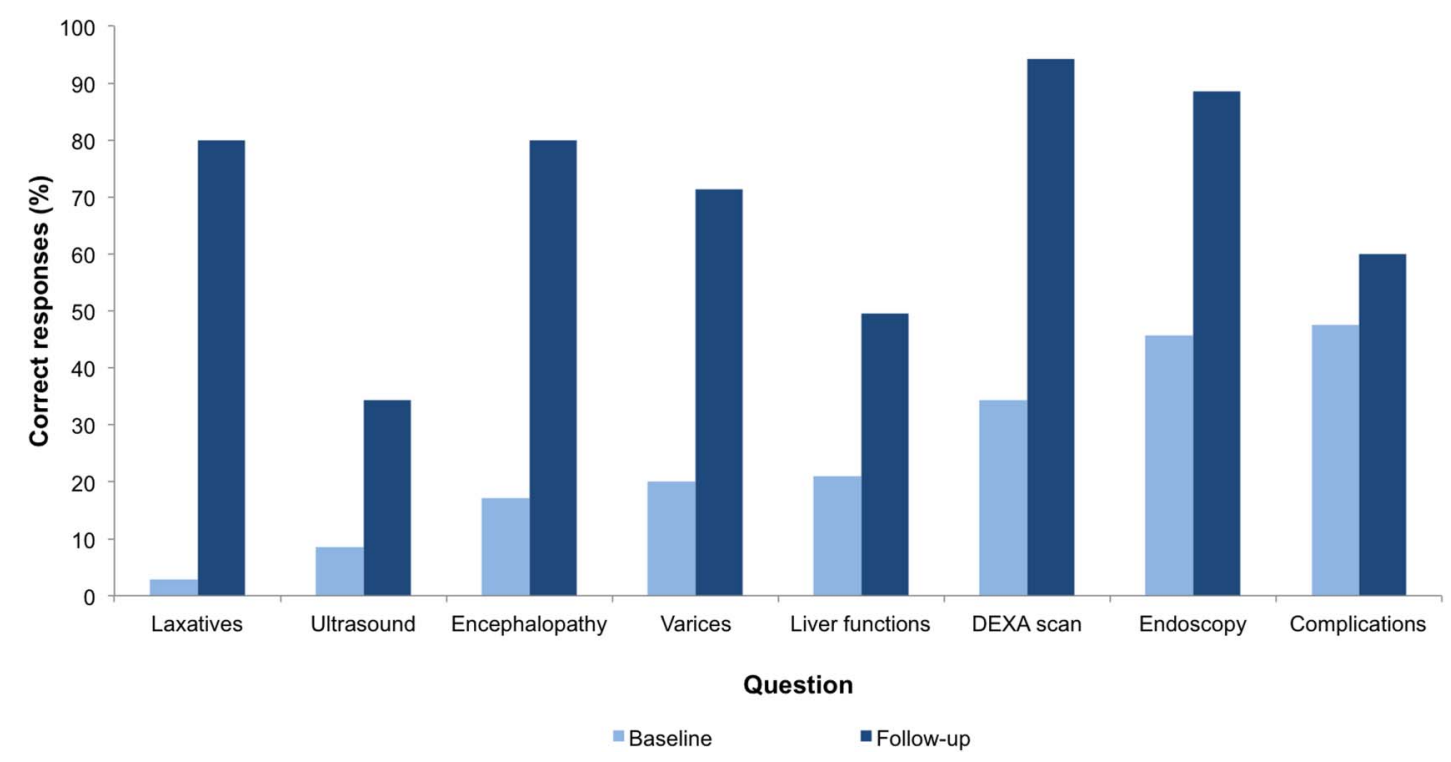

Figure 3 Proportion of responses marked correct for each question on the baseline and follow-up questionnaires ( $n=35)$. DEXA, dual energy $\mathrm{X}$-ray absorptiometry.

$(n=9)$ achieved the lowest median score of $16.7 \%$, while patients in the cholestatic liver disease $(n=9)$ and 'other' $(\mathrm{n}=5)$ groups each had the highest median score of $41.7 \%$.

\section{Follow-up}

Follow-up questionnaires were completed by 35 patients $(67.3 \%)$. The characteristics of this groupalong with those of patients who did not return the follow-up questionnaire-are detailed in table 1.
At baseline, the median questionnaire score of these 35 participants was $25.0 \%$ (IQR $16.7-41.7 \%$ ). This increased to $66.7 \%$ (IQR 50.0-75.0\%) at follow-up, representing a statistically significant improvement of $41.7 \%(\mathrm{p}<0.001)$. Evaluating the whole cohort of 52 patients according to the principles of intention-to-treat analysis (in which no change in score was assumed for those lost to follow-up), a significant overall improvement in median score remained $(33.3 \%, \mathrm{p}<0.001)$. 
A breakdown of scores at baseline and follow-up for each question is shown in figure 3. Knowledge of the reason for having regular ultrasound scans improved by $25.7 \%(\mathrm{p}=0.004)$, regular endoscopies by $42.9 \%(\mathrm{p}<0.001)$, bone mineral density scans by $60.0 \%(\mathrm{p}<0.001)$, being prescribed laxatives by $77.1 \%(\mathrm{p}<0.001)$, risk of variceal bleeding by $51.4 \%$ $(\mathrm{p}<0.001)$, liability to develop encephalopathy by $62.9 \% \quad(\mathrm{p}<0.001)$, knowledge of complications (muscle wasting, impaired coagulation and liver cancer) by $12.4 \%(\mathrm{p}=0.067)$ and knowledge of liver functions by $28.6 \%(\mathrm{p}=0.001)$.

\section{Screencast evaluation}

The screencast was evaluated by 63 individuals, comprising 49 patients and a further 14 relatives or supporters who accompanied them. Of these, $83 \%$ rated the screencast as 'very useful'; $92 \%$ rated the information as 'all relevant' or 'mostly relevant'; $84 \%$ said they would be keen to watch this or similar videos on the internet, and $87 \%$ said they were 'keen' or 'very keen' to have a good knowledge of their liver condition, although $16 \%$ said that knowing this information made them feel scared.

\section{DISCUSSION}

The patients participating in this study had previously been seen in a liver clinic where information about cirrhosis is regularly delivered by healthcare professionals and where information leaflets are readily available. Despite this, baseline understanding was poor. Indeed, only half the patients who had previously undergone an endoscopy were able to identify the reason for this procedure. For liver ultrasound scanning, the figure was only $10 \%$. This is despite the requirement for informed consent in advance of these procedures. Furthermore, almost $10 \%$ of the patients approached in clinic did not recognise that they had cirrhosis. These findings support the hypothesis that patients are not sufficiently informed about their condition.

When education was delivered by screencast, a large increase in knowledge questionnaire scores was observed at follow-up, which took place after an interval of at least one month. This suggests that this form of information delivery enabled sustained retention of new knowledge. Of course, within the interval period, patients may have taken the opportunity to seek out information about their condition from other sources, which may have contributed to the observed increase in knowledge scores. From a pragmatic perspective, this is not overly concerning. Indeed, the experience of viewing the screencast may have empowered individuals to take action in order to develop their understanding of cirrhosis. There were, however, 17 patients (33\%) who did not respond to the follow-up questionnaire who may not have felt engaged with this educational process. These individuals were younger than those who responded to the follow-up questionnaire and the distribution of cirrhosis aetiologies was different, with greater proportions having alcohol-related liver disease and cholestatic liver disease (table 1). Interestingly, this group actually achieved a higher median score on the baseline questionnaire than those who responded to follow-up $(41.7 \%$ vs $25.0 \%)$. Having a reasonable level of knowledge to begin with may therefore be one explanation as to why some patients did not engage with the latter part of this study.

Regarding the viewer evaluation, strongly positive feedback was received regarding the format and usefulness of the screencast. It was notable however that $16 \%$ of participants felt scared by the information. This illustrates the potential for harm in giving information to patients, which should be an important consideration when implementing new educational strategies. In the context of this study, patients viewed the screencast immediately prior to a clinic appointment, which provided them with an opportunity to address their concerns with an experienced professional.

Overall, the results presented here demonstrate that patient knowledge of cirrhosis was substantially improved following the use of a simple, low-cost, multimedia resource. Presenting information in this form may therefore be a useful way for clinicians to facilitate partnership working. Patients may also benefit from the better clinical outcomes associated with increased engagement: Serper et $a l^{10}$ found that liver transplant patients with better knowledge of their medication regimens were more likely to adhere to treatment and less likely to require readmission to hospital. A further study by Singal et $a l^{11}$ found that patients with cirrhosis who felt involved in their care were more likely to attend for HCC screening. Further efforts to educate patients as to the purpose and value of HCC surveillance may help to enhance this service, which has recently been evaluated as requiring substantial development in order to improve outcomes for patients with HCC. ${ }^{12}$ In light of the rising prevalence of chronic liver disease, the work presented here will be extended to generate empirical evidence as to whether multimedia education improves factors such as HCC screening attendance, medication adherence and their associated health outcomes. This work will involve a larger patient cohort and evaluate the persistence of improved patient knowledge over a longer time period.

\section{CONCLUSION}

This study demonstrated that patient understanding of liver cirrhosis is poor. The delivery of relevant information by screencast led to a significant improvement in patients' knowledge about their condition. We therefore present an effective way to empower patients with chronic liver disease with accurate, 
up-to-date and retainable information that could easily be translated to many other chronic disease conditions and contribute to the improvement of a wide range of healthcare services.

\section{Significant of this study}

\section{What is already known on this topic?}

- For effective partnership working in clinical practice, patient knowledge and understanding of their condition is essential.

- There is scarce evidence regarding patient understanding of liver cirrhosis and the effectiveness of current educational strategies.

\section{What this study adds?}

- The baseline knowledge of a cohort of patients with liver cirrhosis was poor, despite regular contact with specialist healthcare providers and surveillance procedures.

- A short educational screencast led to a significant and sustained improvement in knowledge about the management and complications of this disease.

\section{How might it impact on clinical practice in the foreseeable future?}

- Implementation of education by screencast as an adjunct to current strategies could improve patient understanding, and hence their ability to participate in clinical decision-making and undertake self-management.

- This is a simple approach to patient education that could be rapidly adopted in other centres and easily translated to many other conditions.

Acknowledgements The authors thank all of the patients who participated in and evaluated this study. They also thank Geoff Cross for his help in producing the screencast, the outpatient department staff members at St James's University Hospital for their support during the study and the team of reviewers who provided valuable guidance on the content and presentation of the screencast.

Contributors MAG and WF conceived and designed the study; collected, analysed and interpreted the data; drafted the manuscript; approved the final version; and contributed equally. HT analysed and interpreted the data, critically revised the manuscript and approved the final version. MAA, IAR and
RLJ designed the study, critically revised the manuscript and approved the final version.

Competing interests None declared.

Provenance and peer review Not commissioned; externally peer reviewed.

Open Access This is an Open Access article distributed in accordance with the Creative Commons Attribution Non Commercial (CC BY-NC 4.0) license, which permits others to distribute, remix, adapt, build upon this work noncommercially, and license their derivative works on different terms, provided the original work is properly cited and the use is non-commercial. See: http://creativecommons.org/licenses/by$\mathrm{nc} / 4.0 /$

\section{REFERENCES}

1 Williams R, Aspinall R, Bellis M, et al. Addressing liver disease in the UK: a blueprint for attaining excellence in health care and reducing premature mortality from lifestyle issues of excess consumption of alcohol, obesity, and viral hepatitis. Lancet 2014;384:1953-97.

2 D’Amico G, Garcia-Tsao G, Pagliaro L. Natural history and prognostic indicators of survival in cirrhosis: a systematic review of 118 studies. J Hepatol 2006;44:217-31.

3 Coulter A, Ellins J. Effectiveness of strategies for informing, educating, and involving patients. BMJ 2007;335:24-7.

4 Barello S, Graffigna G, Vegni E, et al. The challenges of conceptualizing patient engagement in health care: a lexicographic literature review. J Participat Med 2014;6:e9.

5 Fagerlin A, Sepucha KR, Couper MP, et al. Patients' knowledge about 9 common health conditions: the DECISIONS survey. Med Decis Making 2010;30(5 Suppl):35S-52S.

6 McCartney M. Patient information leaflets: "a stupid system". BMJ 2013;347:f4748.

7 Valery PC, Powell E, Moses N, et al. Systematic review: unmet supportive care needs in people diagnosed with chronic liver disease. BMJ Open 2015;5:e007451.

8 Volk ML, Fisher N, Fontana RJ. Patient knowledge about disease self-management in cirrhosis. Am J Gastroenterol 2013;108:302-5.

9 Davis TC, Berkel HJ, Arnold CL, et al. Intervention to increase mammography utilization in a public hospital. J Gen Intern Med 1998;13:230-3.

10 Serper M, Patzer RE, Reese PP, et al. Medication misuse, nonadherence, and clinical outcomes among liver transplant recipients. Liver Transpl 2015;21:22-8.

11 Singal AG, Volk ML, Rakoski MO, et al. Patient involvement in healthcare is associated with higher rates of surveillance for hepatocellular carcinoma. J Clin Gastroenterol 2011;45:727-32.

12 Cross TJS, Villaneuva A, Shetty S, et al. A national survey of the provision of ultrasound surveillance for the detection of hepatocellular carcinoma. Frontline Gastroenterol 2016;7:82-9. 